\title{
ÉDITORIAL RÉFLEXIONS GÉNÉRALES SUR LA QUALITÉ DE L'EAU DANS LES ÉTABLISSEMENTS DE SANTÉ
}

\author{
J.-C. LABADIE* et P. PARNEIX** \\ Centre de Coordination de Lutte contre les Infections Nosocomiales du Sud-Ouest \\ (CCLIN Sud-Ouest)
}

\section{INTRODUCTION}

La consommation d'eau dans les hôpitaux est particulièrement importante puisqu'elle varie en fonction de la taille de l'établissement de 200 à 1000 litres par lit et par jour. L'hôpital est généralement le plus gros consommateur d'eau dans sa commune. Les usages de l'eau à l'Hôpital sont variés qu'ils soient alimentaires, techniques, sanitaires ou médicaux. Pour chaque usage il convient de disposer d'une eau répondant à des critères physico-chimiques et microbiologiques précis. C'est alors que se pose la question de la qualité de l'eau mise à la disposition des usagers des établissements de santé.

\section{Première partie}

La maîtrise de la qualité de l'eau utilisée à l'hôpital nous oblige à nous poser un certain nombre de questions:

- De quelle eau s'agit-il?

- A quoi va-t-elle servir?

- Qu'est-ce-qui garantit la qualité de l'eau?

* Maître de Conférence des Universités - Praticien Hospitalier.

** Praticien Hospitalier. 
A ces questions nous apportons des réponses qui vont permettre, au fil de l'eau, de trouver des éléments de réflexion aux questions concernant la qualité de l'eau. En effet, il existe un grand nombre de textes réglementaires divers qui permettent aux responsables hospitaliers de mettre en œuvre dans le cadre de la loi des dispositions techniques permettant de garantir en première intention une certaine qualité de l'eau. Il ne s'agit pas là d'une réelle démarche de mise en œuvre d'une assurance de la qualité, mais la connaissance et le respect de ces textes doivent permettre l'acquisition d'un niveau minimal de sécurité dans la prise en charge de la gestion de l'eau dans les établissements de santé. Pour une plus grande facilité dans l'exposé nous allons suivre, d'une certaine façon, le parcours de l'eau en milieu hospitalier.

Nous préciserons, à chaque étape les dispositions réglementaires qui sont en cause.

\section{Deuxième partie}

1 - L'eau du réseau de la ville qui arrive à l'hôpital. Sa qualité est garantie par la loi au titre du Code de la Santé Publique (livre 1, titre 1) mais aussi la loi 92-3 du 3 janvier 1992 sur l'eau et surtout le décret $n^{\circ}$ 89-3 du 3 Janvier 1989 modifié relatif aux eaux destinées à la consommation humaine. Tous ces textes fixent les critères de potabilité que doit remplir l'eau livrée par les collectivités territoriales ou les compagnies fermières aux établissements de santé comme à tous les consommateurs. Le Directeur de l'établissement doit faire effectuer plusieurs analyses de potabilité de l'eau par an.

2 - L'eau destinée à la consommation humaine que l'on stocke à l'hôpital. Les établissements de santé sont obligés de disposer de réserves d'eau égale à au moins une journée de consommation. Pour cela, ils utilisent des bâches qu'ils doivent obligatoirement vidanger, nettoyer et désinfecter une fois par an (article 7.2 du Règlement Sanitaire Départemental). Le nettoyage de ces bâches fait l'objet de recommandations techniques dans la circulaire DGS/VS $n^{\circ} 97482$ du 7 Juillet 1997 et la désinfection de ces réservoirs doit être réalisée conformément aux dispositions de la circulaire du Ministére de la Santé du 15 Mars 1962 (annexe B).

3 - Si l'établissement dispose de ressources privées comme des puits, des forages ou des sources, celles-ci ne peuvent être considérées comme potables et ne peuvent donc être utilisées que pour des usages industriels ou agricoles. Le règlement Sanitaire Départemental fixe les conditions d'exploitation de ces différents ouvrages (articles 10 et 11 ).

Comme on le voit la qualité de l'eau qui entre à l'hôpital dépend en partie du respect de la réglementation en vigueur.

\section{Troisième partie}

La distribution de l'eau à l'hôpital 
A partir des arrivées d'eau, celle-ci est distribuée dans tous les bâtiments et à l'intérieur de ceux-ci grâce à un réseau.

La conception initiale de celui-ci et en particulier l'absence de bras morts est un gage de maintien de la qualité de l'eau. Le choix des matériaux, la lutte contre la corrosion, la mise en place de disconnecteurs et de suppresseurs sont des obligations techniques pour assurer une bonne longévité au réseau.

Le détartrage, voire la désinfection de certaines parties de celui-ci pour éliminer des biofilms sont à l'origine de manœuvres souvent difficiles. Dans ce cas, la qualité dépend des choix initiaux, de la qualité des matériaux mais aussi d'un équilibre acido-basique et minéral de l'eau qui évite que celle-ci n'agresse trop rapidement les conduits. Pour le réseau, la qualité procède avant tout de choix et de mesures techniques adaptées.

\section{Quatrième partie}

Les différents usages de l'eau à l'hôpital

Eau alimentaire ou de boisson : la qualité requise est fixée dans le Décret $n^{\circ}$ 89-3 du 3 Janvier 1989 déjà cité.

Si la distribution d'eau de boisson se fait à partir de fontaines réfrigérées celles-ci doivent faire l'objet d'une maintenance prévue par la circulaire DGS/PGE/ID n ${ }^{\circ} 2068$ du 30 Décembre 1986.

Eau des piscines de rééducation : en l'absence de normes et de textes réglementaires il est conseillé de se référer aux normes d'hygiène applicables aux piscines recevant du public soit le décret n ${ }^{\circ} 81324$ du 7 Avril 1981 modifié.

Eau chaude sanitaire: les risques connus sont liés à l'entartrage du réseau et à la prolifération de certaines bactéries telles les légionnelles et en particulier Legionella pneumophila. Des dispositions réglementaires récentes sont apparues avec la publication de la circulaire DGS n 97/311 du 24 Avril 1997 (annexe 2-3-4) suivie de la circulaire DGS nº 98/771 du 31 décembre 1998.

Eau à usage médical: l'eau pour hémodialyse en est le meilleur exemple. Elle répond à des normes précises fixées par la pharmacopée Européenne et qui détermine les qualités physico-chimiques et microbiologiques que doit présenter ce type d'eau.

L'eau pour le nettoyage et la désinfection des endoscopes représente un autre type d'eau rentrant désormais dans un code normatif. Deux circulaires précisent les exigences requises :

- circulaire DGS/DH n ${ }^{\circ} 236$ du 2 avril 1996 relative aux modalités de désinfection des endoscopes dans les lieux de soins (traitement manuel),

- lettre circulaire DH 987262 du 15 Juillet 1998 concernant la sécurité d'utilisation des dispositifs médicaux (traitement en machines automatiques). 
Ici aussi la qualité de l'eau dans son cadre d'utilisation va dépendre du respect des textes réglementaires qui existent. Ceux-ci imposent en général des seuils qu'il conviendra de respecter.

Pour cela la qualité dépendra non seulement des contrôles effectués et de leur fréquence mais de la mise en place de procédures de contrôle et de maintenance des systèmes qui s'apparentent à la mise en œuvre d'une démarche assurance de la qualité.

L'eau traitée pour les services cliniques: différents traitements physiques ou chimiques sont utilisés pour traiter l'eau destinée à certains usages médicaux, comme le lavage des mains des chirurgiens, les irrigations-lavages en urologie ou l'eau des bains ou douches destinées aux brûlés. Quelque soit le système utilisé il faut savoir que la qualité finale dépend:

- d'un système de traitement fiable,

- de procédures de maintenances cohérentes,

de procédures de contrôles adaptées.

et qu'enfin certains systèmes ont fait la preuve depuis longtemps de leur inefficacité voire de leur dangerosité; il convient alors de les abandonner.

L'eau achetée pour certains usages médicaux : l'exemple en est l'eau pour préparations injectables (EPPI). Les normes sont fixées par la pharmacopée. Il appartient aux responsables des achats de vérifier la conformité.

Comme on peut le voir à l'issue de cette revue des différents usages de l'eau à l'hôpital, il existe un important recueil de textes réglementaires et normatifs. Ceux-ci fixent les seuils, et mettent en œuvre des procédures qui ont pour objet de garantir à l'utilisateur une certaine qualité de l'eau. Le respect de ces dispositions réglementaires s'impose à tous pour obtenir une eau de qualité conforme aux besoins.

\section{CONCLUSION}

Nous la ferons sous la forme de deux questions fondamentales:

1/Avons nous vraiment besoin d'eau pour...?

- humidifier,

a baigner,

- soigner

- mouiller, etc... 
Si la réponse est non alors affranchissons nous des risques liés à l'usage d'une eau plus ou moins douteuse quant au maintien de sa qualité initiale dans le temps.

\section{2/Quelle eau pour quel usage?}

Avons nous fait le bon choix ? en sachant que la qualité de l'acte dépend en partie de la qualité de l'eau.

La réponse préalable à ces deux questions permettra de mieux cerner les risques. Le respect des textes réglementaires et des obligations techniques sera l'amorce d'une démarche qualité.

\section{Résumé}

La qualité de l'eau dans les établissements de santé dépend avant tout du strict respect de la réglementation en vigueur. Celle-ci est abondante et concerne aussi bien la distribution que le stockage et la distribution interne aux établissements.

L'observance des textes réglementaires, la mise en œuvre des dispositions techniques nécessaires, l'établissement de procédures de maintenance des installations, la réalisation des différents contrôles physico-chimiques et microbiologiques prévus par la réglementation sont autant de condition nécessaire à la mise en œuvre d'une démarche qualité. Celle-ci doit permettre de garantir aux usagers un niveau de qualité et donc une sécurité optimale en toutes circonstances.

\section{Summary}

The quality of water in health care centres dépends mainly on the full respect of the legislation on this topic. This specific legislation contains several laws dealing either with water supplies and storage or with hospital pipes.

Observing the staturory regulations, implementing strategies for water supplies maintenance and performing physicochemical and microbiological legal controls are necessary for a correct quality management in order to guarantee a high level of quality of water and on that account a good security for the users. 